\title{
$\mathrm{SnO}$ 的歧化反应对 SnTe 热电性能的优化
}

\author{
胡慧珊，杨君友，辛集武，李思慧，姜庆辉 \\ (华中科技大学 材料科学与工程学院, 材料成型与模具技术国家重点实验室, 武汉 430074)
}

摘 要: $\mathrm{PbTe}$ 基化合物是一种热电性能优良的中温区热电材料, 但铅的毒性限制了其广泛应用, 因此类似化合物 $\mathrm{SnTe}$ 引起了人们关注。但 $\mathrm{SnTe}$ 的载流子浓度较高和晶格热导率较大使其 $Z T$ 值较低。本研究利用 $\mathrm{SnO}$ 歧化反应对 $\mathrm{SnTe}$ 热电性能实现了协同调控。热压烧结过程中 $\mathrm{SnO}$ 在 $500{ }^{\circ} \mathrm{C}$ 左右发生歧化反应生成 $\mathrm{Sn}$ 单质和单分散的 $\mathrm{SnO}_{2}$ 颗粒, $\mathrm{Sn}$ 单质作为自掺杂可以填充 $\mathrm{SnTe}$ 中的 $\mathrm{Sn}$ 空位，导致载流子浓度降低: 相比于 $\mathrm{SnTe}$ 基体, $\mathrm{SnTe}-6 \mathrm{~mol} \% \mathrm{SnO}$ 样品在 $600{ }^{\circ} \mathrm{C}$ 下的电阻率从 6.5 增大到 $10.5 \mu \Omega \cdot \mathrm{m}$, Seebeck 系数从 105 增大到 $146 \mu \mathrm{V} \cdot \mathrm{K}^{-1}$ 。同时, 原位反应生成的 $\mathrm{SnO}_{2}$ 第二相单分散于晶界处, 多尺度散射声子传播而降低晶格热导率, $\mathrm{SnTe}-6 \mathrm{~mol} \% \mathrm{SnO}$ 样品晶格热导率在 $600{ }^{\circ} \mathrm{C}$ 下仅为 $0.6 \mathrm{~W} \cdot \mathrm{m}^{-1} \cdot \mathrm{K}^{-1}$, 相比于基体下降了 $33 \%$ 左右, 从而使 $\mathrm{SnTe}$ 体系的热电性能得到明显提高。最终, 当 $\mathrm{SnO}$ 加 入量为 $6 \mathrm{~mol} \%$ 时, 样品在 $600{ }^{\circ} \mathrm{C}$ 下的 $Z T$ 值 1 , 相比于基体提升了一倍左右。

关 键 词: SnTe; 歧化反应; 热电性能

中图分类号: TB34 文献标识码: A

\section{High Thermoelectric Performance of SnTe from the Disproportionation of SnO}

\author{
HU Hui-Shan, YANG Jun-You, XIN Ji-Wu, LI Si-Hui, JIANG Qing-Hui
}

(State Key Laboratory of Materials Processing and Die \& Mould Technology, School of Materials Science and Engineering, Huazhong University of Science and Technology, Wuhan 430074, China)

\begin{abstract}
PbTe-based compositions are considered as excellent thermoelectric materials for the mid-temperature. However, the toxicity of lead limits its wide application. SnTe compounds, an analogue of $\mathrm{PbTe}$, has attracted much attention. However, its ultrahigh carrier concentration and the large lattice thermal conductivity leads to a low $Z T$ value of SnTe. In this work, the thermoelectric performance of SnTe is synergistically enhanced by introduction of Sn and $\mathrm{SnO}_{2}$ from the disproportionation of $\mathrm{SnO}$ in the process of the hot press sintering. On the one hand, Sn can compensate the Sn vacancies and decrease the carrier concentration of SnTe, leading to a simultaneous enhancement on resistivity and the Seebeck coefficient. For instance, compared with the pristine SnTe, resistivity and the Seebeck coefficient increases from $6.5 \mu \Omega \cdot \mathrm{m}$ to $10.5 \mu \Omega \cdot \mathrm{m}$ and from $105 \mu \mathrm{V} \cdot \mathrm{K}^{-1}$ to $146 \mu \mathrm{V} \cdot \mathrm{K}^{-1}$, respectively, for the sample of SnTe- $6 \mathrm{~mol} \% \mathrm{SnO}$ at $873 \mathrm{~K}$. On the other hand, in-situ generated $\mathrm{SnO}_{2}$ nanoparticles are dispersedly distributed on the grain boundaries, leading to the multiscale phonon scattering and the reduced lattice thermal conductivity. The minimum lattice thermal conductivity value is $0.6 \mathrm{~W} \cdot \mathrm{m}^{-1} \cdot \mathrm{K}^{-1}$ for the sample $\mathrm{SnTe}-6 \mathrm{~mol} \% \mathrm{SnO}$ at $873 \mathrm{~K}$, which is $\sim 33 \%$ reduction compared with that of the pristine SnTe. As a result, the maximum $Z T$ value of $0.96(\sim 100 \%$ enhancement,
\end{abstract}

收稿日期：2018-06-28; 收到修改稿日期：2018-09-11

基金项目: 国家自然科学基金(51811530307, 51772109, 51572098, 51632006); 清华大学新型陶瓷与精细工艺国重实验室开 放基金(KF201704)

National Natural Science Foundation of China (51811530307, 51772109, 51572098, 51632006); Open Fund of State Key Laboratory of New Ceramics and Fine Processing, Tsinghua University (KF201704)

作者简介: 胡慧珊(1994-), 女, 硕士研究生. E-mail: 1724125728@qq.com

通讯作者: 姜庆辉, 副教授. E-mail: qhjiang@hust.edu.cn 
compared with that of the pristine $\mathrm{SnTe}$ ) at $873 \mathrm{~K}$ is achieved for the sample $\mathrm{SnTe}-6 \mathrm{~mol} \% \mathrm{SnO}$.

Key words: SnTe; disproportionation; thermoelectric performance

能源危机正成为全球范围内急需解决的问题, 人们迫切需要寻找一种安全清洁的新能源以满足环 境的可持续发展 ${ }^{[1]}$ 。基于 Seebeck 效应的热电发电 技术具有体积小、轻量、无噪音、可实现热能向电 能的直接转换等优点, 在余热利用领域有着广阔的 应用前景 ${ }^{[2-4]}$ 。热电转换效率取决于无量纲常数 $Z T$ 值, $Z T=\sigma S^{2} T / \kappa$, 其中 $\sigma$ 为电导率, $S$ 为 Seebeck 系数, $\kappa$ 为总热导率, $T$ 为绝对温度 ${ }^{[5]}$ 。为了获得高的 $Z T$, 需 要同时具有高的电导率, 大的 Seebeck 系数和低的 热导率。但是, 由于三种参数间存在强烈的耦合关 系 ${ }^{6]}$, 通常较难获得高的 $Z T$ 值。近年来, 科学工作 者通过能带工程如能带收玫 ${ }^{[7]}$ 和共振能级 ${ }^{[8]}$ 等获得 高的功率因子, 并调控点缺陷、位错和纳米第二相 等显微结构 ${ }^{[9]}$ 降低了晶格热导率, 这种解耦合使得 $Z T$ 得到明显提高。例如, $\mathrm{PbTe}$ 作为典型的中温区热 电材料, $Z T$ 经优化后在 $915 \mathrm{~K}$ 下可达到 $2.2^{[10]}$, 但 $\mathrm{Pb}$ 的毒性限制了其广泛应用。

$\mathrm{SnTe}$ 化合物的熔点为 $1079 \mathrm{~K}$, 空间群为 $F m \overline{3} m$, 室温下块体 $\mathrm{SnTe}$ 的禁带宽度为 $0.18 \mathrm{eV}$, 是一种重 要的窄带隙(直接带隙)半导体, 与 $\mathrm{PbTe}$ 具有相似的 晶体结构和能带结构, 在近红外光探测器、发光二 极管、太阳能电池以及生物医疗等领域有广泛应用 ${ }^{[11]}$ 。 $\mathrm{SnTe}$ 化合物由于存在大量本征 $\mathrm{Sn}$ 空位, 载流子浓 度 $\left(10^{20} \sim 10^{21}\right)$ 较高, Seebeck 系数较低, 电子热导率 较高 ${ }^{[12-13]}$, 并且轻带 $(\mathrm{L}$ 点)和重带 $(\Sigma$ 点)之间存在较大 的能带分离 $(\sim 0.3 \mathrm{eV})$, 严重阻碍了重带对 Seebeck 系 数的贡献 ${ }^{[13-14]}$, 本征 $\mathrm{SnTe}$ 的 $Z T$ 在 $900 \mathrm{~K}$ 下仅为 0.4 左右 ${ }^{[15]}$ 。

国内外若干课题组在 SnTe 化合物方面开展了 大量的工作。Zhao 等 ${ }^{[16]}$ 在( $\left.\mathrm{SnBi}\right) \mathrm{Te}$ 中原位析出 $\mathrm{SrTe}$ 第二相降低声子热导率; $\mathrm{Tan}$ 等 ${ }^{[17]}$ 在 $\mathrm{Sn}_{1.03} \mathrm{Te}$ 中掺 $\mathrm{Cd}$ 进行能带收玫, 并原位引入 $\mathrm{CdS}$ 和 $\mathrm{ZnS}$ 第二相颗粒 散射声子; Pei 等 ${ }^{[18]}$ 在(CuSn) Te 化合物中利用 $\mathrm{Cu}_{2} \mathrm{Te}$ 间隙 $\mathrm{Cu}$ 原子调控晶格热导率, 使 $Z T$ 值达到 1 左右, 不过这种方式只有特定的热电体系和第二相组合 实现了均匀的界面和能量重合的电子带, 不具有普 适性。

本课题组周志伟等 ${ }^{[19]}$ 将 $\mathrm{SnTe}$ 与 $\mathrm{ZnO}$ 纳米颗粒 复合, 通过调控 $\mathrm{n}$ 型 $\mathrm{ZnO}$ 含量优化 $\mathrm{SnTe}$ 热电性能, 取得了较好的效果。但是添加的氧化物是通过化学 法合成, 与 $\mathrm{SnTe}$ 球磨混合, 会造成大量的硬团聚, 理论上对声子的散射贡献不大。本工作通过 $\mathrm{SnO}$ 的
歧化反应, 在 $500{ }^{\circ} \mathrm{C}$ 左右自发析出 $\mathrm{Sn}$ 单质和单分散 的 $\mathrm{SnO}_{2}$ 颗粒，从而提升 $\mathrm{SnTe}$ 的热电性能。

\section{1 实验方法}

\section{1 样品制备}

按化学计量比 $(\mathrm{SnTe})$ 定量称取高纯 $\mathrm{Sn}$ 粉、 $\mathrm{Te}$ 粉, 将其混合均匀后装入石英管中并密封, 然后缓 慢升至 $1123 \mathrm{~K}$ 并保温 $10 \mathrm{~h}$, 随炉冷却至室温。将获 得的熔锭用玛瑙研磨棒碾碎成粉末, 并按摩尔百分 比(SnTe- $x$ mol $\% \mathrm{SnO}, x=0,2,4,6)$ 称取适量 $\mathrm{SnO}$ 粉末 与熔炼合成的 $\mathrm{SnTe}$ 粉末混合均匀。再将混合均匀的 粉末放入充满氩气的不锈钢球磨罐中在 $200 \mathrm{r} / \mathrm{min}$ 转速下球磨 $0.5 \mathrm{~h}$, 球料质量比约为 $20: 1$ 。将球磨 后的粉末置于高纯 $\mathrm{Ar}$ 气保护气氛中进行热压烧结 以获得致密块体, 其中烧结温度为 $823 \mathrm{~K}$, 烧结压力 为 $60 \mathrm{MPa}$ 。

\section{2 表征与测试}

使用 $X$ 射线衍射仪标定样品物相(PANalytical $\chi$ 'Pert PRO, 波长 $0.15418 \mathrm{~nm} 、 \mathrm{Cu}-\mathrm{K} \alpha$ 靶)。使用场 发射扫描电子显微镜(NanoSEM 450) 观察样品的断 口显微形貌。使用 Namicro-III 型热电测试系统系统 测量样品的电阻率和 Seebeck 系数。使用 LFA-427 激光导热仪(NETZSCH, 德国耐驰)测试热扩散系数 $\lambda$, 比热 $\left(C_{\mathrm{P}}\right)$ 取自参考文献值 ${ }^{[20]}$ 。采用阿基米德排水 法测量样品的致密度 $(D)$, 并利用公式 $\kappa=D C_{\mathrm{P}} \lambda$ 计算 得到热导率。使用 STA449F3 (德国耐驰)型同步分析 仪进行热分析, 测试条件为: 升温速率 $10 \mathrm{~K} / \mathrm{min}$ 、 高纯 $\mathrm{Ar}$ 气保护。采用 HMS-5500 型(韩国 Ecopia) 全自动变温霍尔效应测试仪测量样品的霍尔系数。

\section{2 结果与讨论}

\section{1 物相与显微结构}

图 $1 \mathrm{SnO}$ 粉末与热压后样品 $\mathrm{SnTe}-6 \mathrm{~mol} \% \mathrm{SnO}$ 的 DTA 曲线，由 DTA 曲线可见(图右上角 Exo 表示放热 反应), $\mathrm{SnO}$ 粉末在 $725 \mathrm{~K}$ 左右开始发生强烈分解, 并 在 $805 \mathrm{~K}$ 左右达到峰值, 其歧化反应式 ${ }^{[21]}$ 可表示为:

$$
2 \mathrm{SnO}_{(\mathrm{S})}=\mathrm{SnO}_{2(\mathrm{~S})}+\mathrm{Sn}
$$

$\mathrm{SnO}$ 与 $\mathrm{SnTe}$ 粉末混合后热压烧结时, $\mathrm{Sn}$ 单质可 以补充 $\mathrm{Sn}$ 空位实现自掺杂和载流子浓度的优化, $\mathrm{SnO}_{2}$ 相作为析出相可以降低声子传输。热压烧结后 


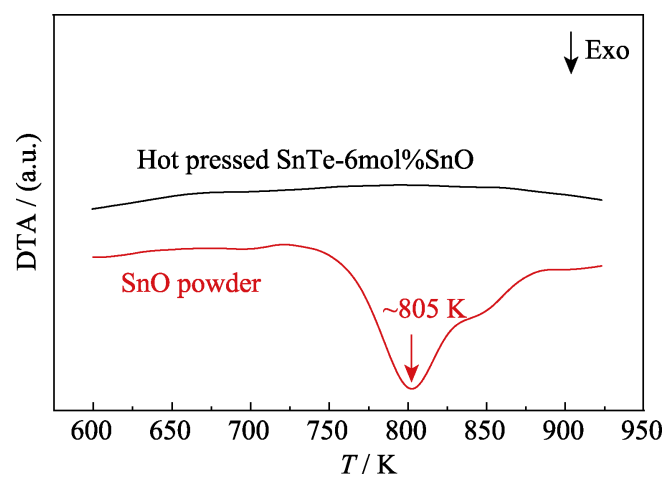

图 $1 \mathrm{SnO}$ 粉末与热压后样品 $\mathrm{SnTe}-6 \mathrm{~mol} \% \mathrm{SnO}$ 的 DTA 曲线 Fig. 1 DTA curves of $\mathrm{SnO}$ powder and hot pressed SnTe-6mol\%SnO

$\mathrm{SnO} / \mathrm{SnTe}$ 块体在相应的温度范围内并未出现任何 放热反应，说明 $\mathrm{SnO}$ 在热压烧结过程中已分解完全, 随后可以实现与 $\mathrm{SnTe}$ 的自掺杂和原位复合。

图 2 为 $\mathrm{SnTe}-x \mathrm{~mol} \% \mathrm{SnO}(x=0,2,4,6)$ 样品的 XRD 图谱, 样品的主衍射峰均与面心立方 SnTe 相 的标准 PDF 卡片(\#46-1210)相对应, 并且样品中存 在 $\mathrm{SnO}_{2}$ 的衍射峰, 其强度随 $\mathrm{SnO}$ 添加量的增大而 增强, 并未探测到任何 $\mathrm{SnO}$ 衍射峰, 这也说明 $\mathrm{SnO}$ 已完全发生歧化反应转变为 $\mathrm{Sn}$ 和 $\mathrm{SnO}_{2}$ 两相。 $\mathrm{SnTe}$ 基体中也含有极少量 $\mathrm{SnO}_{2}$, 这是由于 $\mathrm{SnTe}$ 粉末在 制备过程中会不可避免地存在微量氧化。由高角度 XRD 峰局部放大图(图 1(b)) 可知, 衍射峰随着 $\mathrm{SnO}$ 添加量的增加逐渐向低角度方向偏移, 这是由于 $\mathrm{SnO}$ 分解出的 $\mathrm{Sn}$ 单质与 $\mathrm{SnTe}$ 反应引发晶格发生膨 胀造成的。

图 3(a) (b) 是基体 $\mathrm{SnTe}$ 样品和 $\mathrm{SnTe}-6 \mathrm{~mol} \% \mathrm{SnO}$ 样品的断口 FESEM 照片, 基体中无明显 $\mathrm{SnO}_{2}$ 第二 相存在，而添加 $6 \mathrm{~mol} \% \mathrm{SnO}$ 的 $\mathrm{SnTe}$ 样品晶粒表面 存在部分浅色纳米颗粒。由图 3(c)EDS 分析表明这 些纳米颗粒的组成元素为 $\mathrm{Sn}$ 和 $\mathrm{O}$, 原子比接近 $1: 2$,

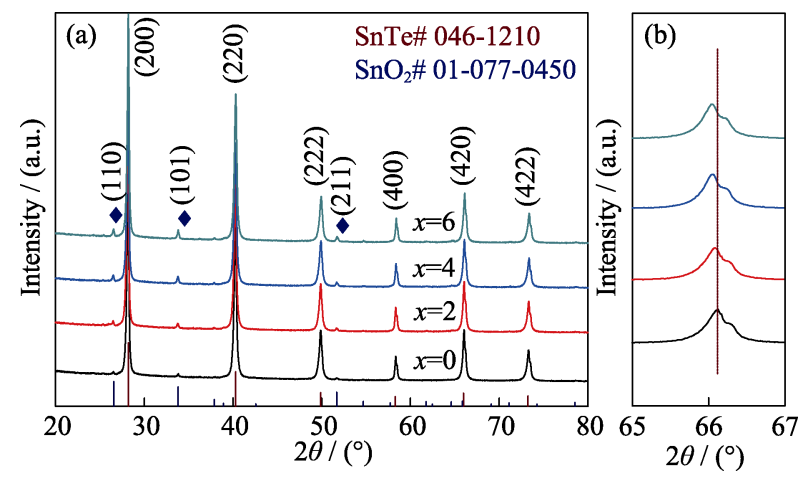

图 $2 \mathrm{SnTe}-x \mathrm{~mol} \% \mathrm{SnO}$ 样品的 XRD 图谱(a)和高角度峰位置 比较(b)

Fig. 2 XRD patterns of SnTe- $x \mathrm{~mol} \% \operatorname{SnO}(x=0,2,4,6)$ samples (a) and high angle peak position comparison between different samples (b)

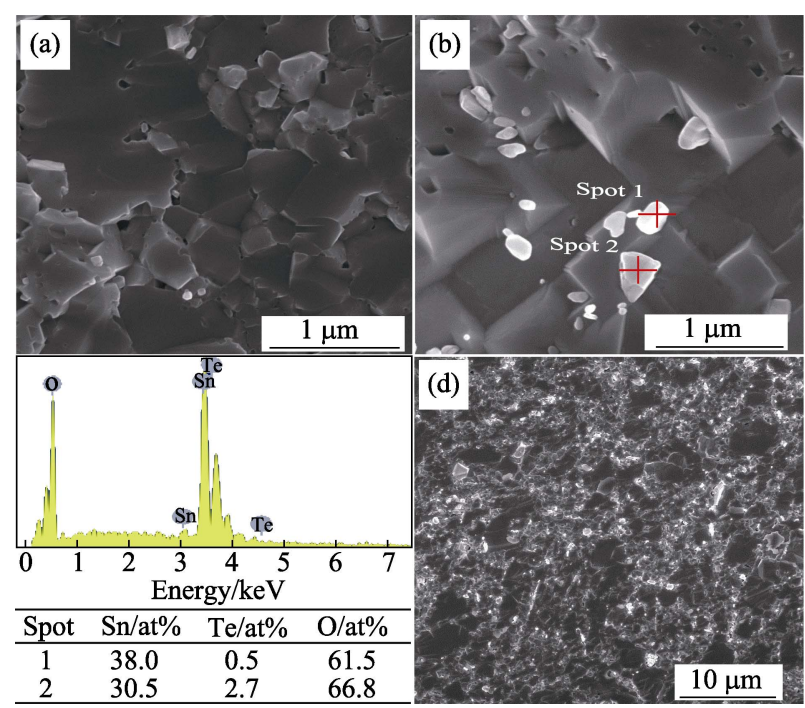

图 $3 \mathrm{SnTe}(\mathrm{a})$ 和 $\mathrm{SnTe}-6 \mathrm{~mol} \% \mathrm{SnO}(\mathrm{b})$ 样品的高倍断口 FESEM 照片, 图(b)中对应区域的 EDS 点扫描(c)和样品 SnTe- $6 \mathrm{~mol} \% \mathrm{SnO}$ 的低倍断口 FESEM 照片(d)

Fig. 3 High magnification cross-sectional FESEM image of (a) $\mathrm{SnTe}$ and (b) SnTe-6mol\%SnO sample, (c) EDS composition analysis results for the spot marked in Fig. 3(b), low magnification cross-sectional FESEM image of SnTe-6mol\%SnO sample (d)

说明第二相为 $\mathrm{SnO}_{2}$, 这与前边 XRD 分析结果相一 致。SnTe- $6 \mathrm{~mol} \% \mathrm{SnO}$ 样品的低倍断口扫描电镜照片 (图 3(d)) 可以观察到 $\mathrm{SnO}_{2}$ 第二相均匀分布在晶界处, 直径从几十到几百纳米不等。

\section{2 电输运性能}

为了研究 $\mathrm{SnO}_{2}$ 第二相单独对 $\mathrm{SnTe}$ 电输运性能 的影响, 首先制备仅添加 $\mathrm{SnO}_{2}$ (与 $\mathrm{SnO}$ 含量相对应) 的 SnTe 样品, 其电阻率和 Seebeck 系数如图 4 所示。 所有样品的电阻率均随温度的升高而变大。

同时, $\mathrm{SnO}_{2}$ 作为第二相会对载流子传输产生额 外散射, 导致样品电阻率随其含量略有增加, 不过 增幅不大; Seebeck 系数基本上不受 $\mathrm{SnO}_{2}$ 含量变化 的影响。整体来说, $\mathrm{SnO}_{2}$ 第二相对 $\mathrm{SnTe}$ 的电输运性 能无明显影响。

与此对应, $\mathrm{SnO}$ 作为前驱体进行歧化反应同时 进行 $\mathrm{Sn}$ 的自掺杂和 $\mathrm{SnO}_{2}$ 的复合，导致 $\mathrm{SnTe}$ 复合热 电材料的电输运性能发生了明显变化, $\mathrm{SnTe}$ 体系的 电阻率随 $\mathrm{SnO}$ 含量增加而变大(图 5(a) 所示)。如图 5(b) 所示, 样品的室温载流子浓度随 $\mathrm{SnO}$ 添加量增大而 显著降低。这说明 $\mathrm{SnO}$ 歧化反应析出的 $\mathrm{Sn}$ 单质对 $\mathrm{SnTe}$ 进行了有效的自掺杂, Sn 空位浓度显著降低, 导致 $\mathrm{p}$ 型载流子浓度降低、迁移率提高。

图 5(c) 是不同样品的 Seebeck 系数随温度的变化 曲线, 其现象比较反常: $600 \mathrm{~K}$ 以下随 $\mathrm{SnO}$ 添加量增 大而降低, $600 \mathrm{~K}$ 以上反而随 $\mathrm{SnO}$ 添加量增大而升 

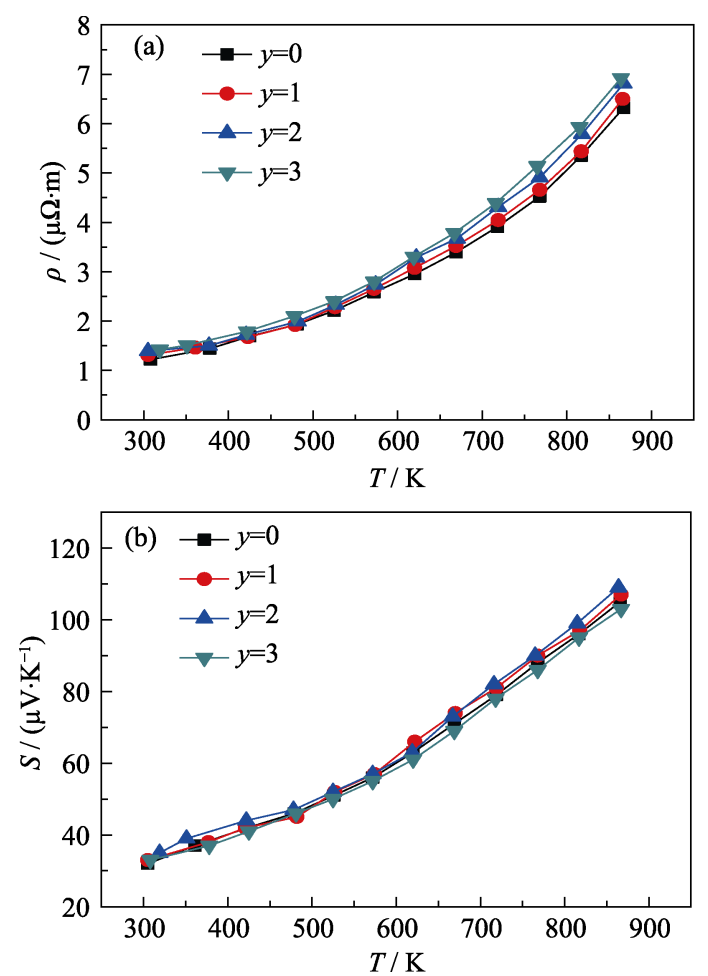

图 $4 \mathrm{SnTe}-y \mathrm{~mol} \% \mathrm{SnO}_{2} \quad(y=0,1,2,3)$ 样品的电阻率 (a) 和 Seebeck 系数(b)随温度变化的关系

Fig. 4 Temperature dependence of (a) electrical resisitivity and (b) Seebeck coefficient of SnTe-ymol $\% \mathrm{SnO}_{2}(y=0,1,2,3)$ samples

高, 这与 $\mathrm{SnTe}$ 单独掺杂 $\mathrm{Sn}$ 元素的相关研究报导非 常类似 ${ }^{[17]}$ 。这种现象可能与 SnTe 独特的能带结构
有关: SnTe 存在轻带和重带两个非简并价带 ${ }^{[13-14]}$,

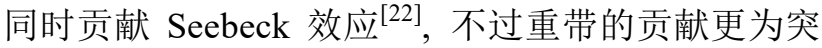
出。室温下, 样品的载流子浓度随 $\mathrm{Sn}$ 掺杂量增加而 更低, 费米能级更高, 从而靠近轻带 ${ }^{[23]}$, 使有效质 量变小。而有效质量对 Seebeck 系数的影响大于载 流子浓度变化对 Seebeck 系数的影响, 因此在低温 段, 样品的 Seebeck 系数随 SnO 添加量降低; 在高 温段, 样品由于热激发导致重带也参与电输运 ${ }^{[22]}$, 在轻带和重带都参与电输运的情况下, 样品的有效 质量接近甚至超过基体样品，同时载流子浓度降低 导致 Seebeck 系数随 $\mathrm{SnO}$ 添加量增加而增大。600 K 应为样品载流子浓度与有效质量两因素对 Seebeck 系数影响的平衡点。

图 5(d) 展示了 $\mathrm{SnTe}-x \mathrm{~mol} \% \mathrm{SnO}$ 样品的功率因子 随温度变化的关系。基于 Seebeck 系数随 $\mathrm{SnO}$ 添加 量的温度变化规律, 样品的功率因子在低温段随 $\mathrm{SnO}$ 添加量增加而下降, $750 \mathrm{~K}$ 以上随 $\mathrm{SnO}$ 添加量 增加而显著升高。当 $\mathrm{SnO}$ 添加量为 $4 \mathrm{~mol} \%$ 时样品功 率因子在 $873 \mathrm{~K}$ 下达到 $2123 \mathrm{~mW} \cdot \mathrm{m}^{-1} \cdot \mathrm{K}^{-2}$, 相比基体 $\left(1708 \mathrm{~mW} \cdot \mathrm{m}^{-1} \cdot \mathrm{K}^{-2}\right)$ 提升了 $24.3 \%$ 。

\section{3 热输运性能}

图 6 总结了 $\mathrm{SnTe}-x \mathrm{~mol} \% \mathrm{SnO}$ 样品的总热导率、 电子热导率和晶格热导率随温度变化的规律, 其中 电子热导率由维德曼-弗兰兹(Wiedemann-Franz)定 律 $\kappa_{\mathrm{e}}=L \sigma T$ 计算出, 其中 $L$ 为洛伦兹常数, $\sigma$ 为电导率,
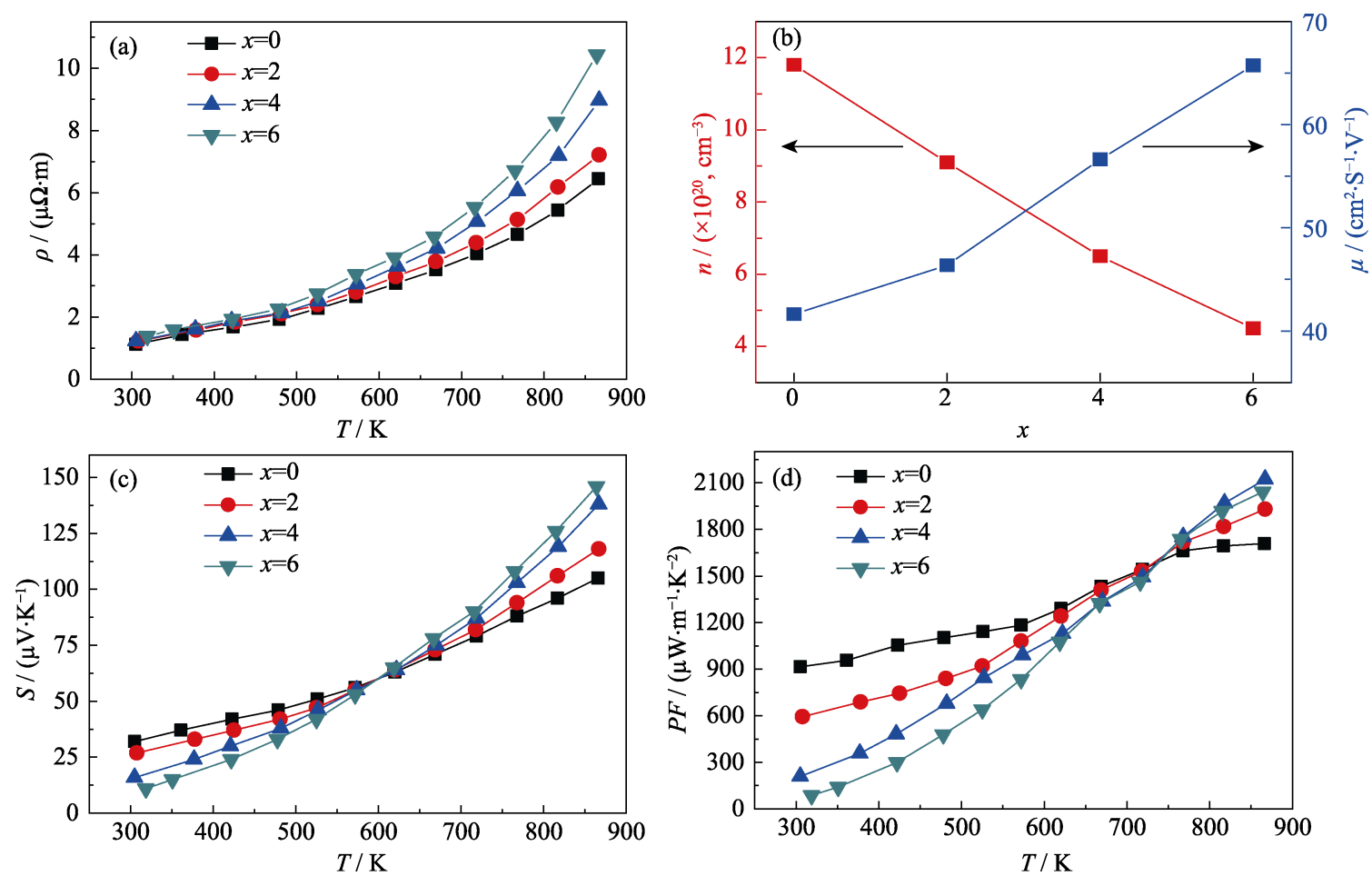

图 $5 \mathrm{SnTe}-x \mathrm{~mol} \% \mathrm{SnO}$ 样品的电阻率(a), 室温载流子浓度和迁移率(b), Seebeck 系数(c)和功率因子(d)随温度变化的关系

Fig. 5 Temperature dependence of (a) electrical resisitivity, (b) Seebeck coefficient, (c) power factor of $\mathrm{SnTe}-\mathrm{xmol}_{\mathrm{m}} \mathrm{SnO} \mathrm{S}_{2}$ $(x=0,2,4,6)$ samples, $(d)$ carrier concentration $(n)$ and carrier mobility $(m)$ at room temperature of the samples 

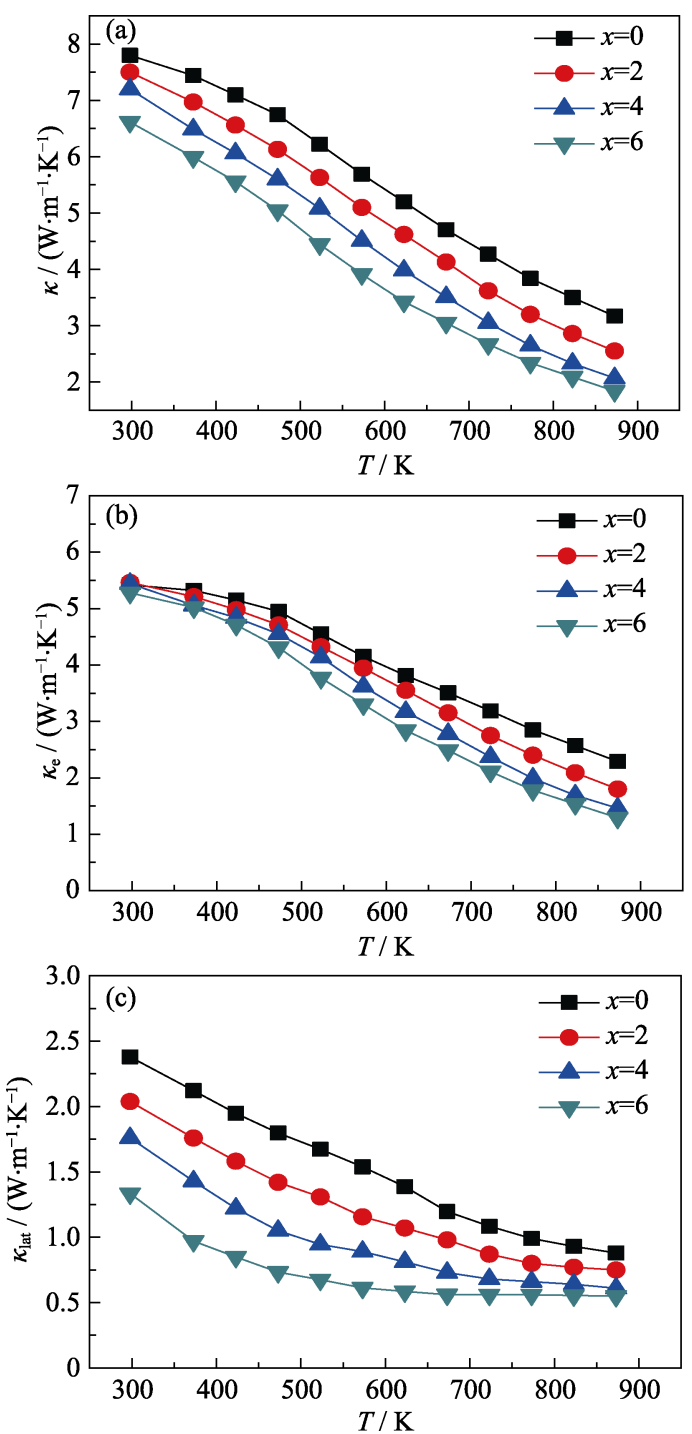

图 $6 \mathrm{SnTe}-x \mathrm{~mol} \% \mathrm{SnO}$ 样品的(a)热导率, (b)电子热导率和(c) 晶格热导率随温度变化的关系

Fig. 6 Temperature dependence of (a) thermal conductivity, (b) carrier thermal conductivity and (c) lattice thermal conductivity of $\mathrm{SnTe}-x \mathrm{~mol} \% \mathrm{SnO}$ samples

$T$ 为绝对温度, 这里 $L$ 按照单带模型 Seebeck 系数计 算得到 ${ }^{[24]}$, 总热导率减去电子热导率即为晶格热导 率。Pei 等 ${ }^{[18]}$ 和 Tan 等 ${ }^{[25]}$ 报道的基体 $\mathrm{SnTe}$ 的晶格热 导率分别约为 $1.7 \mathrm{~W} \cdot \mathrm{m}^{-1} \cdot \mathrm{K}^{-1}(@ 850 \mathrm{~K})$ 和 $1.5 \mathrm{~W} \cdot \mathrm{m}^{-1} \cdot \mathrm{K}^{-1}$ (@873 K), 其制备工艺为熔炼-热压烧结/SPS。本实 验的基体 $\mathrm{SnTe}$ 在 $873 \mathrm{~K}$ 下具有较低的晶格热导率, 这与试样的合成工艺(熔炼-球磨-热压烧结)有关, 即球磨后颗粒较细, 并伴随有大量晶体缺陷产生, 热压后显微结构及缺陷得以保存, 对声子散射作用 增强。Zhang 等 ${ }^{[26]}$ 通过类似方法制备 $\mathrm{SnTe}$ 基体的晶 格热导率 $\left(\sim 1 \mathrm{~W} \cdot \mathrm{m}^{-1} \cdot \mathrm{K}^{-1}(@ 873 \mathrm{~K})\right)$ 与本工作中 $\mathrm{SnTe}$ 基体的晶格热导率值相当。

添加 $\mathrm{SnO}$ 明显降低了 $\mathrm{SnTe}$ 样品的总热导率, 由 $3.2 \mathrm{~W} \cdot \mathrm{m}^{-1} \cdot \mathrm{K}^{-1}$ 降低到 $1.8 \mathrm{~W} \cdot \mathrm{m}^{-1} \cdot \mathrm{K}^{-1}(@ 873 \mathrm{~K})$ 。基于

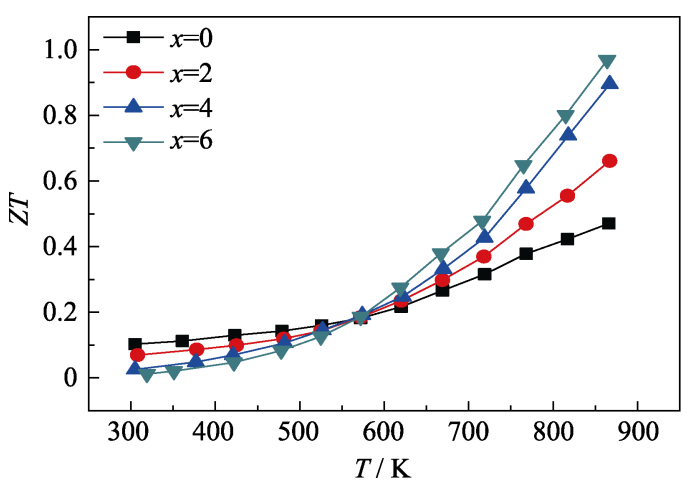

图 $7 \mathrm{SnTe}-x \mathrm{~mol} \% \mathrm{SnO}$ 样品的 $Z T$ 值随温度变化的关系

Fig. 7 Temperature dependence of $Z T$ values of SnTe- $x \mathrm{~mol} \% \mathrm{SnO}$ samples

图 4 分析结果, $\mathrm{SnO}_{2}$ 第二相对样品的电输运性能无 显著影响, 因此图 6(b)样品电子热导率的降低主要 来源于 $\mathrm{SnO}$ 歧化反应导致的 $\mathrm{Sn}$ 自掺杂, 使电子热导 率由 $2.3 \mathrm{~W} \cdot \mathrm{m}^{-1} \cdot \mathrm{K}^{-1}$ 降低到 $1.2 \mathrm{~W} \cdot \mathrm{m}^{-1} \cdot \mathrm{K}^{-1}(@ 873 \mathrm{~K})$ 。 由于 $\mathrm{Sn}$ 含量少幅变化对 $\mathrm{SnTe}$ 的晶格热导率几乎无 影响 ${ }^{[17]}$, 样品的晶格热导率的降低主要来自于 $\mathrm{SnO}$ 歧化反应析出的 $\mathrm{SnO}_{2}$ 第二相对声子的散射作用。基 于图 3 分析讨论, 歧化反应析出的 $\mathrm{SnO}_{2}$ 颗粒均匀弥 散分布在 $\mathrm{SnTe}$ 晶界处且尺寸从数十到数百纳米不 等, 形成多尺度的声子散射中心，从而有效降低样 品的晶格热导率, 使其声子热导率由 $0.9 \mathrm{~W} \cdot \mathrm{m}^{-1} \cdot \mathrm{K}^{-1}$ 降低到 $0.6 \mathrm{~W} \cdot \mathrm{m}^{-1} \cdot \mathrm{K}^{-1}(@ 873 \mathrm{~K})$ 。

\section{4 无量纲热电优值 $(Z T)$}

图 7 为 $\mathrm{SnTe}$ 样品的 $Z T$ 值随温度变化的曲线, 随 $\mathrm{SnO}$ 添加量的增加, 由于歧化反应析出的 $\mathrm{Sn}$ 单 质和 $\mathrm{SnO}_{2}$ 纳米相对样品电热输运性能的解耦合调 控增强, 样品高温段的 $Z T$ 值显著增大。当 $\mathrm{SnO}$ 加 入量为 $6 \mathrm{~mol} \%$ 时, $\mathrm{SnTe}$ 复合热电材料的最大 $Z T$ 值 达到 0.96(@873 K), 相比于基体( 0.47)提升了一倍 左右。

\section{3 结论}

$\mathrm{SnO}$ 在升温过程中会发生歧化反应, 生成 $\mathrm{Sn}$ 单 质和 $\mathrm{SnO}_{2}$ 。本研究利用该反应直接复合 $\mathrm{SnO}$ 和 $\mathrm{SnTe}$ 粉体，在热压烧结过程中 $\mathrm{SnO}$ 原位分解实现 $\mathrm{Sn}$ 的 自掺杂和 $\mathrm{SnO}_{2}$ 纳米相的同步添加。其中 $\mathrm{Sn}$ 的自掺 杂会显著降低载流子浓度及电子热导率, 并提高功 率因子; 原位析出的 $\mathrm{SnO}_{2}$ 纳米相单分散均匀地分 布在 $\mathrm{SnTe}$ 晶界处, 对声子实现多尺度散射, 有效降 低晶格热导率。当 $\mathrm{SnO}$ 添加量为 $6 \mathrm{~mol} \%$ 时, $\mathrm{SnTe}$ 的 $Z T$ 值达0.96(@873 K), 比基体提升一倍左右。这种 
歧化反应的协同调控方式, 具有一定的普适性, 通 过优化参数可以进一步有效提高 $\mathrm{SnTe} 、(\mathrm{BiSb})_{2} \mathrm{Te}_{3}$

和 $\mathrm{SnSe}$ 等若干体系的热电性能。

\section{参考文献:}

[1] 朱铁军, 赵新兵. $\beta-\mathrm{FeSi}_{2}$ 热电材料的性能优化及测试方法. 材 料科学与工程, 1999(4): 55-59.

[2] ZHU T J, CAO Y Q, ZHANG Q, et al. Bulk nanostructured thermoelectric materials: preparation, structure and properties. Journal of Electronic Materials, 2010, 39(9): 1990-1995.

[3] YU C, ZHU T J, SHI R Z, et al. High-performance half-heusler thermoelectric materials $\mathrm{Hf}_{1-x} \mathrm{Zr}_{x} \mathrm{NiSn}_{1-y} \mathrm{Sb}_{y}$ prepared by levitation melting and spark plasma sintering. Acta Materialia, 2009, 57(9): 2757-2764.

[4] XIE H H, WANG H, PEI Y Z, et al. Beneficial contribution of alloy disorder to electron and phonon transport in half-Heusler thermoelectric materials. Advanced Functional Materials, 2013, 23(41): 5123-5130.

[5] CHEN Z W, ZHANG X Y, PEI Y Z, et al. Manipulation of phonon transport in thermoelectrics. Advanced Materials, 2018, 30(17): 1705617-1-12.

[6] MOSHWAN R, YANG L, ZOU J, et al. Eco-friendly SnTe thermoelectric materials: progress and future challenges. Advanced Functional Materials, 2017, 27(43): 1703278-1-18.

[7] AL RAHAL AL ORABI R, MECHOLSKY N A, HWANG J, et al. Band degeneracy, low thermal conductivity, and high thermoelectric figure of merit in SnTe-CaTe alloys. Chemistry of Materials, 2016, 28(1): 376-384.

[8] TAN X F, LIU G Q, XU J T, et al. Thermoelectric properties of In-Hg co-doping in SnTe: energy band engineering. Journal of Materiomics, 2018, 4(1): 62-67.

[9] JIANG Q H, YANG J Y, LIU Y, et al. Microstructure tailoring in nanostructured thermoelectric materials. Journal of Advanced Dielectrics, 2016, 6(1): 1630002-1-16.

[10] BISWAS K, HE J Q, WANG G Y, et al. High thermoelectric figure of merit in nanostructured p-type $\mathrm{PbTe}-\mathrm{MTe}(\mathrm{M}=\mathrm{Ca}, \mathrm{Ba})$. Energy \& Environmental Science, 2011, 4(11): 4675-4684.

[11] HERMAN F, KORTUM R, ORTENBURGER I, et al. Relativistic band structure of $\mathrm{GeTe}, \mathrm{SnTe}, \mathrm{PbTe}, \mathrm{PbSe}$, and $\mathrm{PbS}$. Journal de Physique Colloques, 1968, 29(C4): C4-62-C64-77.

[12] BREBRICK R F. Deviations from stoichiometry and electrical properties in SnTe. Journal of Physics and Chemistry of Solids, 1963, 24(1): 27-36.
[13] BREBRICK R F, STRAUSS A J. Anomalous thermoelectric power as evidence for two-valence bands in SnTe. Physical Review, 1963, 131(1): 104-110.

[14] CROCKER A J, ROGERS L M. Interpretation of the Hall coefficient, electrical resistivity and seebeck coefficient of p-type lead telluride. British Journal of Applied Physics, 1967, 18(5): 563.

[15] VEDENEEV V P, KRIVORUCHKO S P, SABO E P. Tin telluride based thermoelectrical alloys. Semiconductors, 1998, 32(3): 241244.

[16] ZHAO L D, ZHANG X, WU H J, et al. Enhanced thermoelectric properties in the counter-doped SnTe system with strained endotaxial SrTe. Journal of the American Chemical Society, 2016, 138(7): 2366-2373.

[17] TAN G J, ZHAO L D, SHI F Y, et al. High thermoelectric performance of p-type SnTe via a synergistic band engineering and nanostructuring approach. Journal of the American Chemical Society, 2014, 136(19): 7006-7017.

[18] PEI Y Z, ZHENG L L, LI W, et al. Interstitial point defect scattering contributing to high thermoelectric performance in SnTe. Advanced Electronic Materials, 2016, 2(6): 1600019.

[19] ZHOU Z W, YANG J Y, JIANG Q H, et al. Multiple effects of Bi doping in enhancing the thermoelectric properties of SnTe. Journal of Materials Chemistry A, 2016, 4(34): 13171-13175.

[20] ZHENG L L, LI W, LIN S Q, et al. Interstitial defects improving thermoelectric SnTe in addition to band convergence. ACS Energy Letters, 2017, 2(3): 563-568.

[21] 陶东平, 杨显万. 氧化亚锡歧化还原动力学和二氧化锡还原机 理. 中国有色金属学报, 1998(1): 129-133.

[22] PEI Y Z, SHI X Y, LALONDE A, et al. Convergence of electronic bands for high performance bulk thermoelectrics. Nature, 2011, 473(7345): 66.

[23] LITTLEWOOD P B, MIHAILA B, SCHULZE R K, et al. Band structure of SnTe studied by photoemission spectroscopy. Physical Review Letters, 2010, 105(8): 086404-1-4.

[24] LIU W S, ZHANG Q Y, LAN Y C, et al. Thermoelectric property studies on Cu-doped n-type $\mathrm{Cu}_{x} \mathrm{Bi}_{2} \mathrm{Te}_{2.7} \mathrm{Se}_{0.3}$ nanocomposites. $A d$ vanced Energy Materials, 2011, 1(4): 577-587.

[25] TAN G J, SHI F Y, HAO S H, et al. Codoping in SnTe: enhancement of thermoelectric performance through synergy of resonance levels and band convergence. Journal of the American Chemical Society, 2015, 137(15): 5100-5112.

[26] ZHANG Q, LIAO B L, LAN Y C, et al. High thermoelectric performance by resonant dopant indium in nanostructured SnTe. Proceedings of the National Academy of Sciences, 2013, 110(33): 13261-13266. 